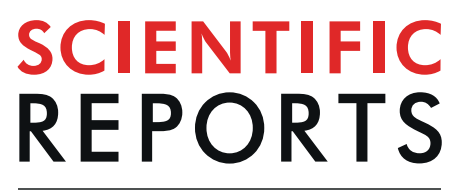

natureresearch

Check for updates

\title{
$56 \mathrm{~nm}$ Wide-Band Tunable
}

\section{Q-Switched Erbium Doped Fiber Laser with Tungsten Ditelluride $\left(\mathrm{WTe}_{2}\right)$ Saturable Absorber}

\author{
Harith Ahmad ${ }^{1,2,3 凶}$, Hissah Saedoon Albaqawi ${ }^{1}$, Norazriena Yusoff ${ }^{1}$ \& Chong Wu Yi ${ }^{1}$
}

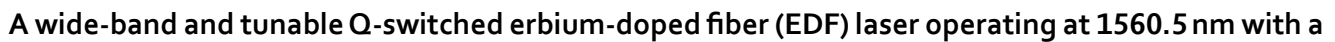
tungsten ditelluride (WTe $\mathrm{W}_{2}$ ) saturable absorber (SA) is demonstrated. The semi-metallic nature of WTe $\mathrm{W}_{2}$ as well as its small band gap and excellent nonlinear optical properties make it an excellent SA material. The laser cavity uses an $89.5 \mathrm{~cm}$ long EDF, pumped by a $980 \mathrm{~nm}$ laser diode as the linear gain while the $\mathrm{WTe}_{2}$ based SA generates the pulsed output. The WTe $\mathrm{T}_{2}$ based SA has a modulation depth, non-saturable loss and saturation intensity of about $21.4 \%, 78.6 \%$, and $0.35 \mathrm{~kW} / \mathrm{cm}^{2}$ respectively. Stable pulses with a maximum repetition rate of $55.56 \mathrm{kHz}$, narrowest pulse width of $1.77 \mu$ s and highest pulse energy of $18.09 \mathrm{~nJ}$ are obtained at the maximum pump power of $244.5 \mathrm{~mW}$. A $56 \mathrm{~nm}$ tuning range is obtained in the laser cavity, and the output is observed having a signal to noise ratio (SNR) of $48.5 \mathrm{~dB}$. The demonstrated laser has potential for use in a large number of photonics applications.

Pulsed lasers are highly desirable laser sources that are typically realized in the form of mode-locked ${ }^{1}$ and Q-switched lasers ${ }^{2}$ that can be further classified as either active ${ }^{3}$ or passive ${ }^{4}$ systems. Active pulsed laser is accomplished through the use of acousto-optic ${ }^{5}$ and electro-optic modulators ${ }^{6}$ among other technique to generate pulses. This approach gives significant control over the various parameters of the generated pulses, but at the cost of a bulky and expensive setup. Normally, passive pulse laser can be typically achieved through the use of saturable absorbers $(\mathrm{SAs})^{7,8}$. This approach, while providing less control over the output parameters of the generated pulses, is advantageous in that it allows for compact and cost-effective fiber laser cavities to be designed ${ }^{9}$. Topological insulators (TIs) such as bismuth selenide $\left(\mathrm{Bi}_{2} \mathrm{Se}_{3}\right)^{10}$, bismuth telluride $\left(\mathrm{Bi}_{2} \mathrm{Te}_{3}\right)^{11}$, black phosphorus ${ }^{12,13}$, perovskite $^{14}$, as well as carbon-based materials such as carbon nanotubes (CNTs) ${ }^{15}$ and graphene ${ }^{16}$ are amongst the various materials that have shown great potential as SAs in order to produce pulses in erbium-doped fiber (EDF) laser cavities. This is because the afore-mentioned groups of materials exhibits a larger nonlinear-optical response ${ }^{17}$ as well as exceptional optical properties that include a large absorption coefficient, low defect density and long carrier lifetimes ${ }^{18}$. Besides that, the thin 2D structure of these materials give it an advantage in terms of unique photonic, magnetic, and electronic properties that is crucial for pulsed laser generation ${ }^{19}$.

Q-switched pulse generation has long been the focus of research efforts due to its advantageous intrinsic characteristics that include long pulses with higher pulse energies and durations ${ }^{20}$, which is highly desirable for applications in material processing, remote sensing, range finding, and medicine ${ }^{21-25}$. Furthermore, Q-switching is easier to induce as compared to mode-locking ${ }^{26}$, which would require delicate balancing between the dispersion and nonlinearities in the laser cavity ${ }^{27}$. As such, Q-switching is generally the preferred method of obtaining pulses in a laser cavity. Recently, researchers have focused their attention towards the exploration of transition metal dichalcogenide (TMD) group of materials in generating Q-switched pulses at broadband wavelengths. As SAs, TMD have significant advantages that include strong absorption ${ }^{28}$, high nonlinear optical response, optical fiber compatibility and ease of fabrication ${ }^{29}$. Luo et al..$^{30}$ demonstrated passively Q-switched Ytterbium (Yb), Erbium (Er) and Thulium (Tm) fiber lasers using a molybdenum disulfide $\left(\mathrm{MoS}_{2}\right)$ film as an SA, and their findings show that few-layer $\mathrm{MoS}_{2}$ films have significant potential as broadband SAs operating at the near to mid-infrared regions. Furthermore, Zhang et al..$^{31}$ demonstrated the use of tungsten disulfide $\left(\mathrm{WS}_{2}\right)$ to passively Q-switch

${ }^{1}$ Photonics Research Centre, University of Malaya, 50603, Kuala Lumpur, Malaysia. ${ }^{2}$ Physics Department, Faculty of Science, University of Malaya, 50603, Kuala Lumpur, Malaysia. ${ }^{3}$ Visiting Professor at the Department of Physics, Faculty of Science and Technology, Airlangga University, Surabaya, 60115, Indonesia. ${ }^{\circledR e}$-mail: harith@um.edu.my 
$\mathrm{EDF}$ and $\mathrm{Yb}$-fiber laser cavities. The $\mathrm{WS}_{2}$ based $\mathrm{SA}$ is capable of generating Q-switched pulses with microsecond durations and kilohertz repetition rates at lasing wavelengths of 1030 and $1558 \mathrm{~nm}$, demonstrating the potential for $\mathrm{WS}_{2}$ as an SA in ultrafast photonic applications.

Tungsten ditelluride $\left(\mathrm{WTe}_{2}\right)$ is another member of the TMD family and is a unique material as it is associated to a rising class of Weyl semimetals. This makes them highly promising for future applications as electronic, spintronic, and optoelectronic devices ${ }^{32,33}$ and also as a potential candidate for quantum spin Hall insulator materials ${ }^{34}$. Even though $\mathrm{WTe}_{2}$ belongs to the TMD family, its uniqueness arises from the additional structural distortion caused by the $\mathrm{W}$ atoms forming zigzag chains in a quasi-one-dimensional arrangement ${ }^{35}$. Furthermore, the small overlap between the valence band and conduction band of $\mathrm{WTe}_{2}$ results in an almost gapless band; as small as $0.7 \mathrm{eV}^{36} \mathrm{com}^{-}$ pared to other TMD materials which typically have band gaps of more than $1 \mathrm{eV}^{37}$. As such, $\mathrm{WTe}_{2}$ is more suitable for applications in near-infrared systems such as photodetectors, communications devices and in the area of ultrafast optics. WTe $\mathrm{W}_{2}$ was also been found to have unique characteristics such as a high unsaturated magneto-resistance (MR) as well as good superconducting behaviour while under high pressure ${ }^{38}$ which makes $\mathrm{WTe}_{2}$ quite attractive for nanoelectronic applications ${ }^{39}$. In addition to that, the very fast relaxation of photocarriers makes WTe $\mathrm{S}_{2}$ suitable for generating Q-switched pulses ${ }^{38}$. In a study conducted by Wang et. al. ${ }^{40}$, an ultrafast pulse was successful generated in a mode-locked thulium-doped fiber laser through the use of magnetron-sputtering deposited WTe $\mathrm{W}_{2}$ as an SA. Stable soliton pulses with pulse durations of $1.25 \mathrm{ps}$ and average output powers of $39.9 \mathrm{~mW}$ were obtained at a central wavelength of $1915.5 \mathrm{~nm}$. Similarly, Koo et. al. ${ }^{41}$, developed passively mode-locked ultrafast lasers at $1556.2 \mathrm{~nm}$ using defective, bulk-structured WTe ${ }_{2}$ microflakes as an SA. They discovered that the structural dimensionality does not critically influence the saturable absorption performance of $\mathrm{WTe}_{2}$.

In this work, $\mathrm{WTe}_{2}$ is demonstrated as a wideband SA in a passively Q-switched EDFL operating at the 1.5 $\mu \mathrm{m}$ region. The $\mathrm{WTe}_{2}$ SA has a modulation depth of $21.4 \%$ and saturation intensity of $\sim 0.35 \mathrm{~kW} / \mathrm{cm}^{2}$. Stable Q-switched pulses are obtained at a central lasing wavelength of $1560.5 \mathrm{~nm}$, which can be tuned over a range of $56 \mathrm{~nm}$ from $1522 \mathrm{~nm}$ to $1578 \mathrm{~nm}$. The proposed SA would have significant benefits and potential to be used in various applications.

\section{Characterization of a Tungsten Ditelluride (WTe2) - Based SA}

Figure 1(a) shows the morphology of the WTe $\mathrm{W}_{2}$ layer as obtained from a Hitachi SU8220 field-emission scanning electron microscope (FESEM) under x13k magnification. From the figure, it can be seen that the WTe ${ }_{2}$ nanoparticles have a flake-like morphology, and tend to stack together to form thicker flakes. Figure 1(b) shows the energy dispersive X-ray (EDX) profile of the $\mathrm{WTe}_{2}$ sample, with signal peaks associated only with the tungsten (W) and tellurium (Te) elements observed. This shows both the formation of $\mathrm{WTe}_{2}$ sample as well as its purity. The inset of Fig. 1(b) shows the area of the $\mathrm{WTe}_{2}$ sample from which the scan was obtained, as well as the weight and atomic percentage of the two elements which are $0.91 \%$ and $42.15 \%$, for $\mathrm{W}$ and $0.87 \%$ and $57.85 \%$ for Te respectively.

An NT MDT atomic force microscope (AFM) is used to measure the thickness of the $\mathrm{WTe}_{2}$ film. The location of the $\mathrm{WTe}_{2}$ film's surface that was chosen for the thickness measurement is denoted by the blue line. The obtained AFM image is shown in Fig. 1(c), and it can be seen that the $\mathrm{WTe}_{2}$ sample used in this work consists of about six individual layers stacked together. Figure $1(\mathrm{~d})$ gives the height profile of $\mathrm{WTe}_{2}$ film projected within the blue line. Six steps can be counted on the height profile, which gives the estimated thickness of the $\mathrm{WTe}_{2}$ film to be six layers. The green line represents the position of one $\mathrm{WTe}_{2}$ layer, with the thickness of a single layer estimated to be about $35.6 \mathrm{~nm}$. As such, the overall thickness of the sample is estimated to be $213.6 \mathrm{~nm}$. Further characterization of the $\mathrm{WTe}_{2}$ sample is also carried out using a Renishaw inVia Raman microscope linked with a $532 \mathrm{~nm}$ line from a doubled Nd:YAG laser as the excitation source. As displayed in Fig. 1(e), two distinct Raman peaks are seen in the Raman spectrum of WTe $e_{2}$. The peaks located at 163 and $211 \mathrm{~cm}^{-1}$ can be assigned to the in-plane $\mathrm{A}_{1}{ }^{7}$ and $\mathrm{A}_{1}{ }^{9}$ modes of $\mathrm{WTe}_{2}$, respectively ${ }^{42,43}$.

The SA assembly is formed by sandwiching a small piece of the $\mathrm{WTe}_{2}$-PVA between two optical fiber patchcords. A small amount of index matching gel is placed on the surface of the optical fiber patchcord, on which the $\mathrm{WTe}_{2}$-PVA piece is then placed. Using a fiber adaptor, another patchcord is joined to the first, thus forming the SA assembly. A white light source is linked to the SA as to obtain the linear optical transmission characteristics of the $\mathrm{WTe}_{2}$ film. The optical spectrum is observed from $1200 \mathrm{~nm}$ to $1600 \mathrm{~nm}$ using a Yokogawa AQ6370C optical spectrum analyser (OSA) with an average transmission (T) of $92.64 \%$ observed at $1560 \mathrm{~nm}$ as in Fig. 2(a). The nonlinear optical absorption characteristic of the $\mathrm{WTe}_{2} \mathrm{SA}$ are investigated using the balanced twin detector technique, with a Menlo Systems ELMO femtosecond erbium laser with a $100 \mathrm{MHz}$ repetition rate and 2.88 ps pulse width at $1564 \mathrm{~nm}$ serving as the signal source. The obtained data is inserted into the saturation model equation ${ }^{44}$ :

$$
\alpha(I)=\frac{\alpha_{s}}{1+I / I_{s a t}}+\alpha_{n s}
$$

where $\alpha_{s}, I, I_{s a t}$, and $\alpha_{n s}$ is refer to the modulation depth, input intensity, saturation intensity, and non-saturable loss respectively. The relative parameters for the $\mathrm{WTe}_{2} \mathrm{SA}$ are obtained based from the resulting fitted curve as given in Fig. 2(b), with the modulation depth, saturation intensity and non-saturable loss of the $\mathrm{WTe}_{2} \mathrm{SA}$ being $\sim 21.4 \%$, $0.35 \mathrm{~kW} / \mathrm{cm}^{2}$, and $78.6 \%$, respectively. The insertion loss of the $\mathrm{WTe}_{2} \mathrm{SA}$ is measured to be approximately $0.31 \mathrm{~dB}$.

\section{Experimental Setup}

Figure 3 shows the schematic of the passively Q-switched ring cavity EDF laser. The laser cavity uses a $980 \mathrm{~nm}$ laser diode (LD) as the pump source with a maximum output power of $244.5 \mathrm{~mW}$ and injected into the cavity via the $980 \mathrm{~nm}$ port of a $980 / 1550 \mathrm{~nm}$ wavelength-division multiplexer (WDM). The output of the WDM is connected to a $89.5 \mathrm{~cm}$ long EDF which has a dopant concentration, absorption, mode field diameter, and numerical aperture of $2000 \mathrm{ppm}, 16 \mathrm{~dB} / \mathrm{m}$ at $1530 \mathrm{~nm}, 9.5 \mu \mathrm{m}$ at $1550 \mathrm{~nm}$ and 0.13 , respectively. The EDF serves as the gain 

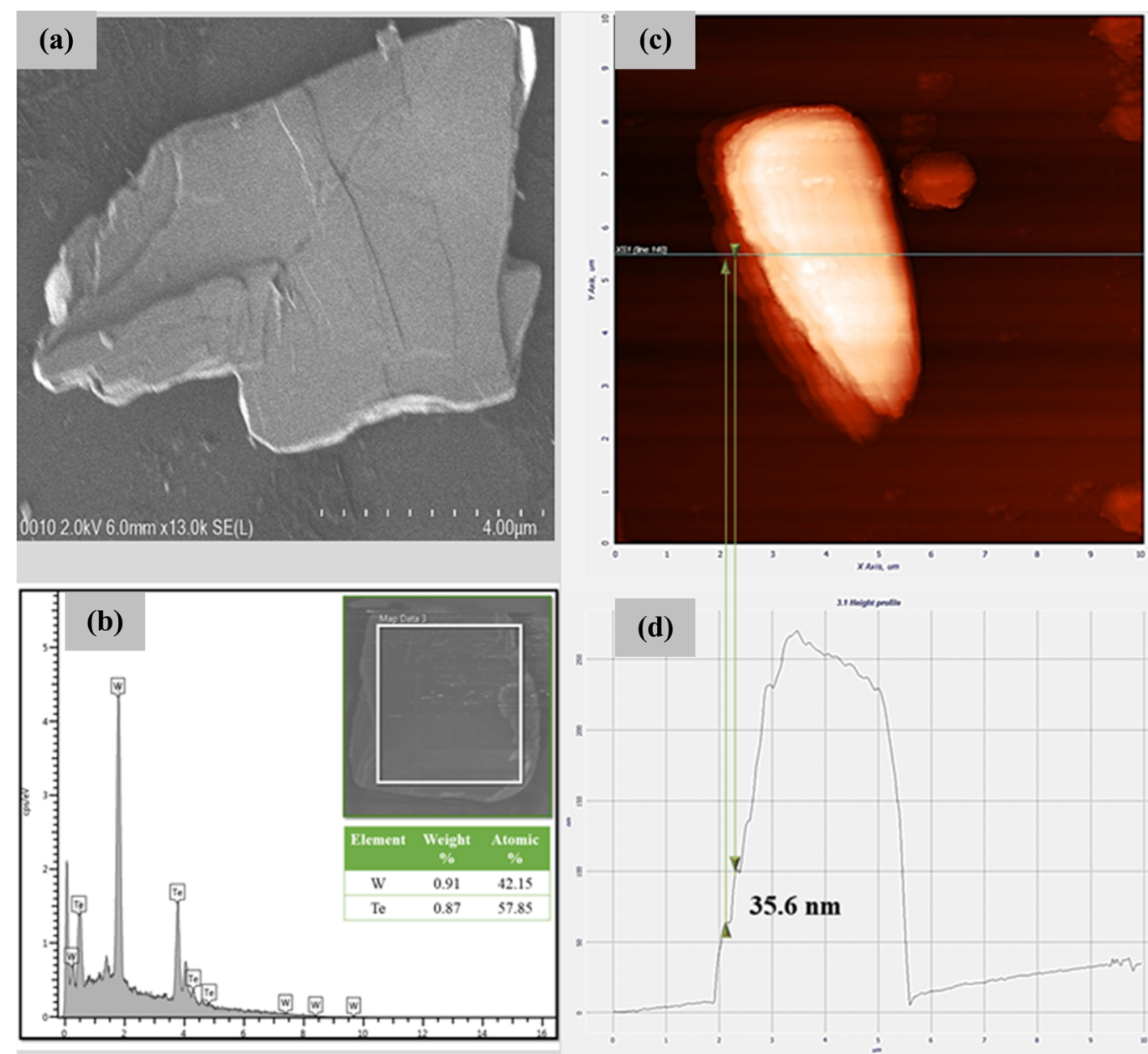

(d)

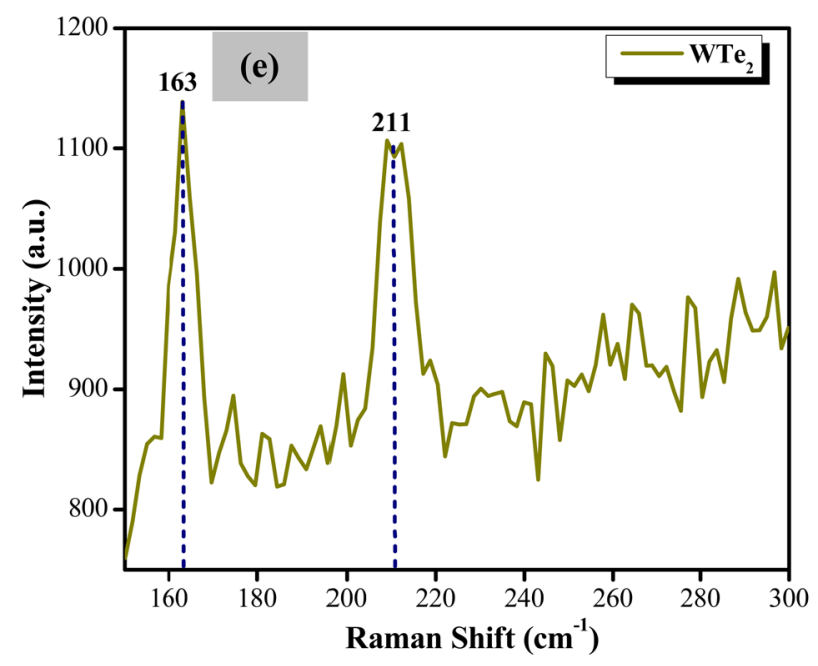

Figure 1. (a) FESEM image, (b) EDX spectrum, (c) AFM image, (d) Lateral height measurement, and (e) Raman spectrum for few layers $\mathrm{WTe}_{2}$.

media, and is connected to an optical isolator as well as an optical tunable bandpass filter (TBPF) with a tuning range of $80 \mathrm{~nm}$ and a resolution of $1 \mathrm{~nm}$. The signal now reaches the WTe $\mathrm{W}_{2}$ based SA, and an 80:20 optical coupler is used to obtain $20 \%$ of the signal. The $80 \%$ port of the coupler is connected to the $1550 \mathrm{~nm}$ port of the WDM, thereby closing the optical cavity. Polarization controllers (PCs) are not included in the design of the cavity as Q-switched pulses can be observed immediately with the incorporation of the $\mathrm{WTe}_{2}$ based SA into the laser cavity and without any further optimization of the propagating signal. Therefore, adding a PC would not improve the performance of the cavity, and will instead induce additional insertion losses to the cavity. 

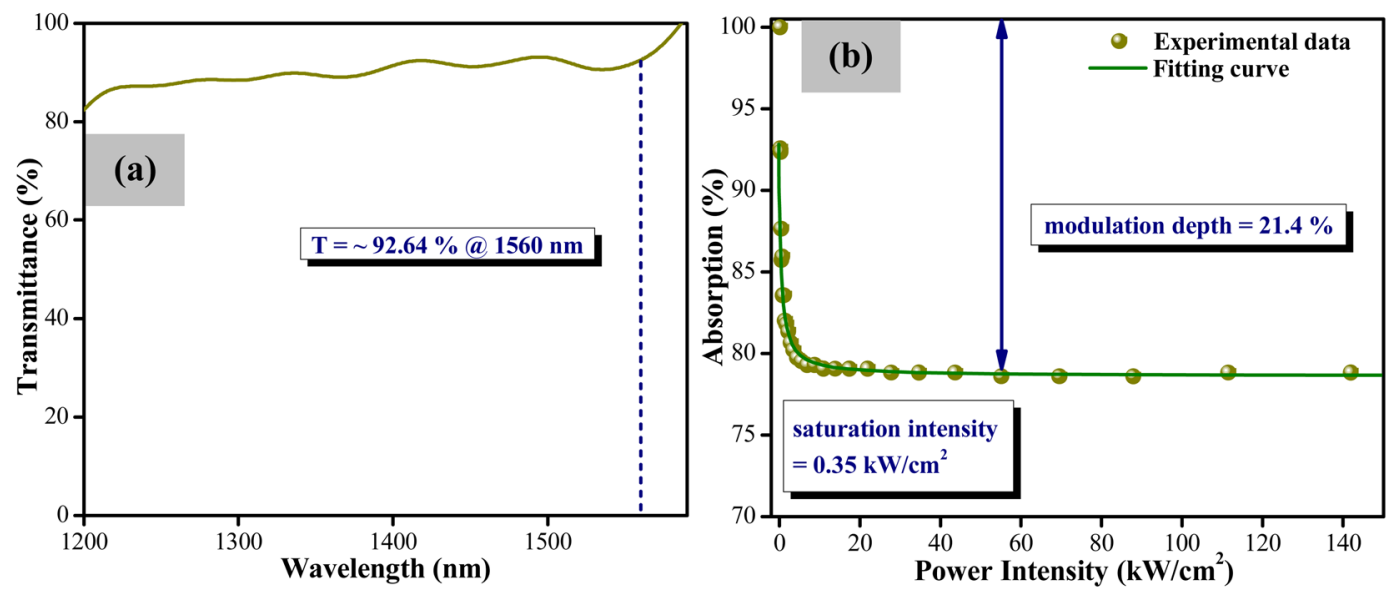

Figure 2. (a) Linear optical transmission and (b) Nonlinear optical absorption of $\mathrm{WTe}_{2}$-PVA film.

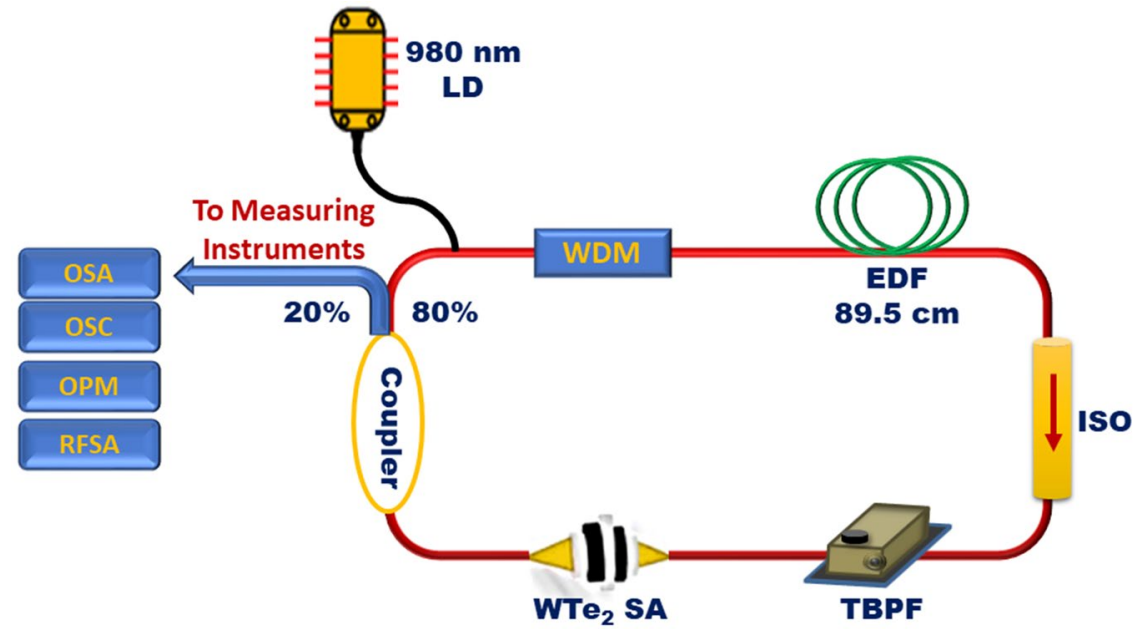

Figure 3. Schematic illustration of the experimental setup for generating EDF laser with the assistance of WTe SA. (LD: laser diode; WDM: wavelength division multiplexer; EDF: Erbium-doped fiber; ISO: isolator; $\mathrm{WTe}_{2}$ SA: Tungsten ditelluride saturable absorber; OSA: optical spectrum analyzer; OSC: oscilloscope; OPM: optical power meter; RFSA: radio frequency spectrum analyzer; TBPF: tunable optical bandpass filter).

\section{Results and Discussion}

When operating without the $\mathrm{WTe}_{2} \mathrm{SA}$, no pulsed outputs can be detected, thus confirming that the generation of pulses is due solely to the SA and not arising from other optical phenomena. With the WTe $\mathrm{SA}_{2}$ in the cavity, continuous wave $(\mathrm{CW})$ operation is obtained at a threshold pump power of $77.43 \mathrm{~mW}$ while Q-switching begins at a threshold pump power of $124.9 \mathrm{~mW}$. Pulsing is observed to continue steadily until the maximum pump power of $244.5 \mathrm{~mW}$ is reached. The pulse characteristics of the Q-switched EDF laser at a pump power of $244.5 \mathrm{~mW}$ are shown in Fig. 4. The optical spectrum of the pulses is given in Fig. 4(a), where the central wavelength is determined to be $1560.5 \mathrm{~nm}$ with a $3-\mathrm{dB}$ band width of $1.55 \mathrm{~nm}$. The oscilloscope trace of the Q-switched pulses is given in Fig. 4(b), whereby the generated pulses have a peak-to-peak pulse interval of $18.86 \mu$ s that corresponds to a repetition rate of $55.56 \mathrm{kHz}$. In Fig. 4(c), the single pulse profile has a full width at half maximum (FWHM) duration of $1.77 \mu \mathrm{s}$. Based on the RF spectrum, a frequency of $55.56 \mathrm{kHz}$ is observed in Fig. 4(d), with the pulses haveing an average signal-to-noise ratio (SNR) of about $48.5 \mathrm{~dB}$. This indicates that the generated Q-switched pulses are highly stable, and comparable to that of other similar systems ${ }^{45,46}$.

Figure 5(a) shows the oscilloscope traces of the output pulses obtained at four different pump powers. It can be seen from the figure that the repetition rate increases while the spacing between pulses decreases as the pump power is increased. These trends are characteristic of Q-switching operation. The repetition rate and pulse width as a function of pump power is given in Fig. 5(b) and shows the repetition rate increasing from $38.39 \mathrm{kHz}$ to $55.56 \mathrm{kHz}$ when the pump power is increased from $124.9 \mathrm{~mW}$ to $244.5 \mathrm{~mW}$. At the same time, the pulse width decreases from $3.67 \mu \mathrm{s}$ to $1.77 \mu \mathrm{s}$, with a sharp decrease in the pulse width before a pump power of $236.5 \mathrm{~mW}$ and a slower decrease after it. The slower decrease of the pulse width at the higher pump powers is attributed to the change in the saturable-absorption property of the $\mathrm{WTe}_{2} \mathrm{SA}$ from parasitic continuous waves that can partially bleach $i^{47}$. As shown in Fig. 5(c), the variation of the output power and pulse energy against the pump power 

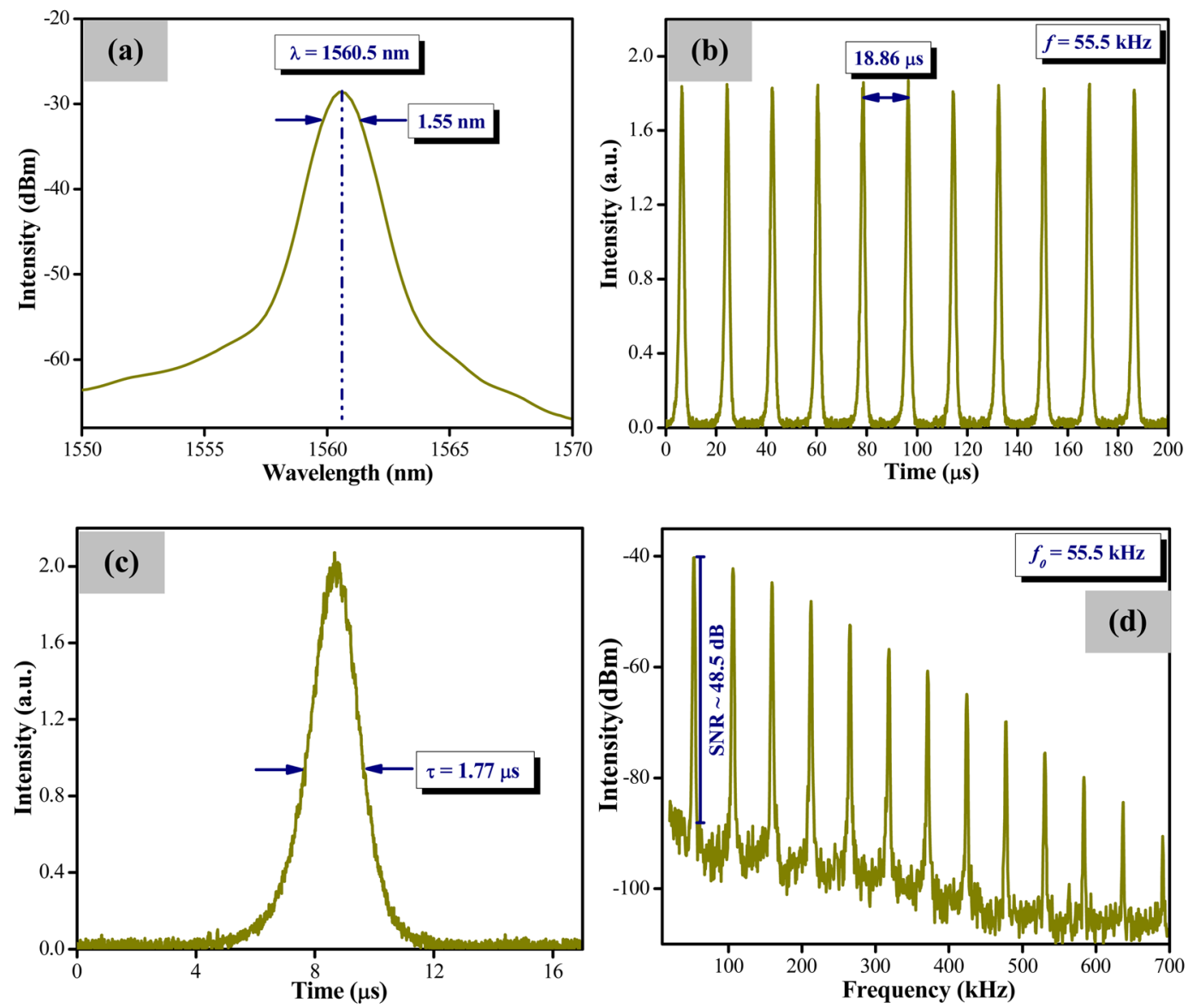

Figure 4. (a) Optical spectrum of the laser output, (b) pulse train (c) single pulse profile and (d) RF spectrum. All measurements are taken at a pump power of $244.5 \mathrm{~mW}$ :.

shows a generally rising trend, rising from $0.28 \mathrm{~mW}$ to $1.01 \mathrm{~mW}$ and $7.31 \mathrm{~nJ}$ to $18.09 \mathrm{~nJ}$, respectively as the pump power is pushed to the maximum. However, as can be seen from the figure, a decrease in the average output power is observed at a pump power of $204.8 \mathrm{~mW}$, before the average output power continues to rise normally against the increasing pump power. This sudden decrease in power is a result of a kink in the output power of the $\mathrm{LD}$ as the drive current increases. This causes the output power from the fiber laser to drop slightly, then continue to increase normally as the drive current increases. Another possibility as put forward by $\mathrm{Adel}^{48}$ is that when the drive current increased, this causes an increase in the temperature of the laser diode, and shifts the lasing wavelength away from the peak absorbing wavelength of $974 \mathrm{~nm}$. This reduces the absorption occurring in the EDF, thus lowering the output power of the fiber laser. Further increasing the drive current of the LD will increase the pump power and cause the output power of the fiber laser to increase in tandem, as would be expected.

Figure 6 gives the RF spectra as captured over a span of 60 minutes at a pump power of $221 \mathrm{~mW}$. No significant changes in the SNR value over the time period can be observed, with the average SNR value being $48.5 \mathrm{~dB}$. Based on the inset of Fig. 6, no frequency drifting can be observed with the frequency remaining constant at about $\sim 53.8 \mathrm{kHz}$ throughout the observation period. These results indicate the high stability of the proposed laser and also that there is no significant degradation of the $\mathrm{WTe}_{2} \mathrm{SA}^{\prime}$ 's performance throughout its operation period.

Figure 7(a) shows the optical spectra of the tunable wavelength output obtained at a constant pump power of $194.2 \mathrm{~mW}$. From the figure, no Q-switching operation can be observed below the wavelength of $1522 \mathrm{~nm}$ or beyond the wavelength of $1578 \mathrm{~nm}$, giving the laser a tuning wavelength of $56 \mathrm{~nm}$. Figure 7 (b) shows the repetition rates against different wavelengths, and from the figure it can be seen that there is a gradual increase in the repetition rate as the wavelength is tuned from 1522 to $1532 \mathrm{~nm}$. In general, the high repetition rate at the larger gain region of the cavity is due to lower cavity losses. This happens as a result of the more rapid bleaching of the $\mathrm{SA}$ which is due to faster population inversion/depletion rates ${ }^{49}$. Above a wavelength of the $1532 \mathrm{~nm}$, the variation of repetition rate trend follows the amplified spontaneous emission (ASE) spectrum of the laser, as the inset of Fig. 7(b), from which the EDF's gain profile is obtained ${ }^{50}$. The variation of the pulse width of the Q-switched laser output over the same wavelength tuning range is given in Fig. 7(c). From the figure, it can be seen that the pulse width increases from $1.3 \mu$ s to $2.6 \mu$ s over the increasing wavelength range. This is attributed to the different wavelength regions experiencing different gains, and as such variations in the repetition rate and pulse width that correspond to the gain curve of the cavity.

A comparison of the passively Q-switched fiber laser in this work against other similar systems using different $\mathrm{SAs}$ is given in Table 1. From the table, it can be seen that the proposed laser of this work using the WTe ${ }_{2}-\mathrm{PVA}$ 

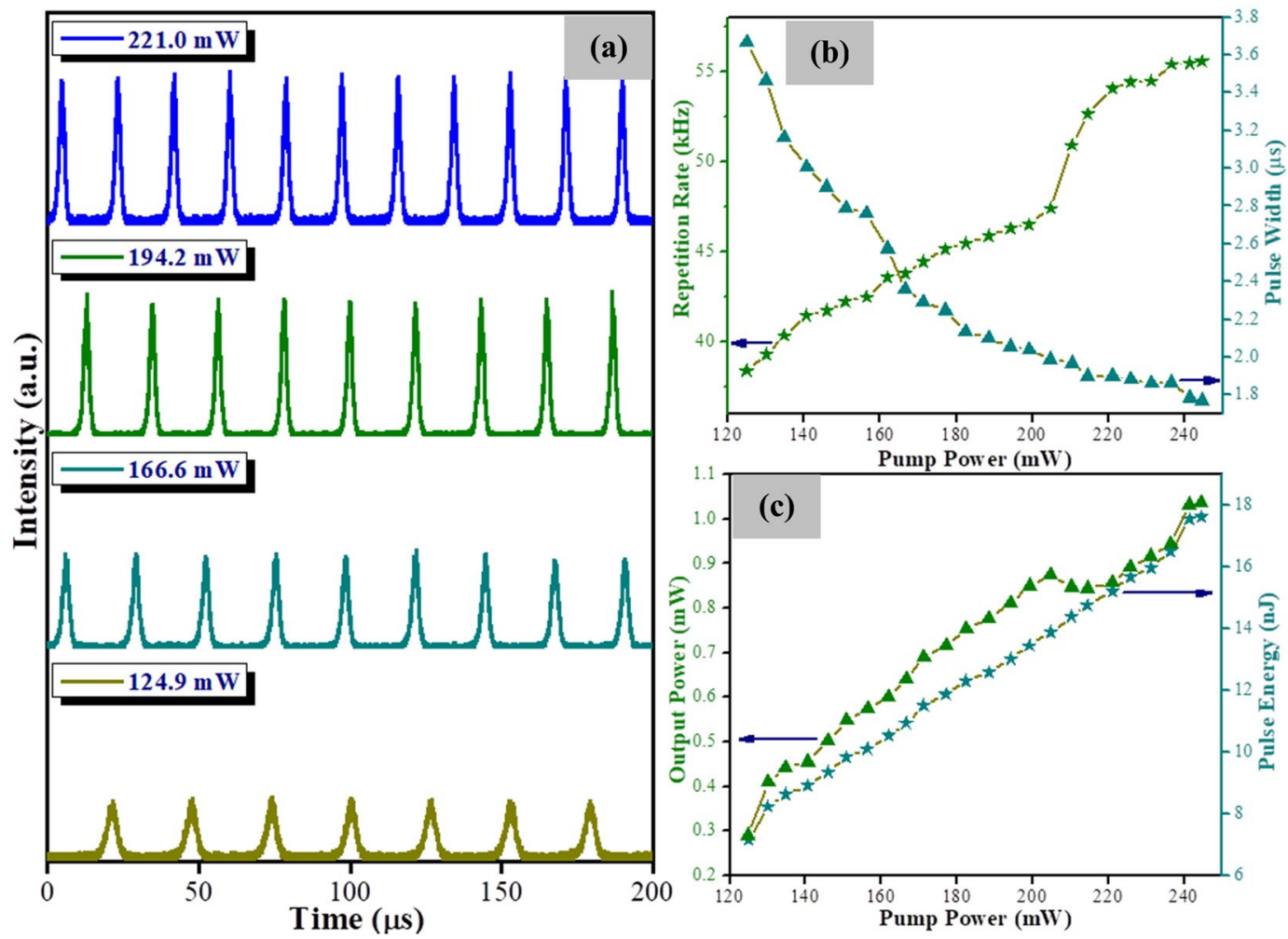

Figure 5. (a) Passively Q-switched pulse trains at the pump powers of $124.9 \mathrm{~mW}, 166.6 \mathrm{~mW}, 194.2 \mathrm{~mW}$, and $221 \mathrm{~mW}$, respectively, (b) repetition rate and pulse width and (c) output power and pulse energy.

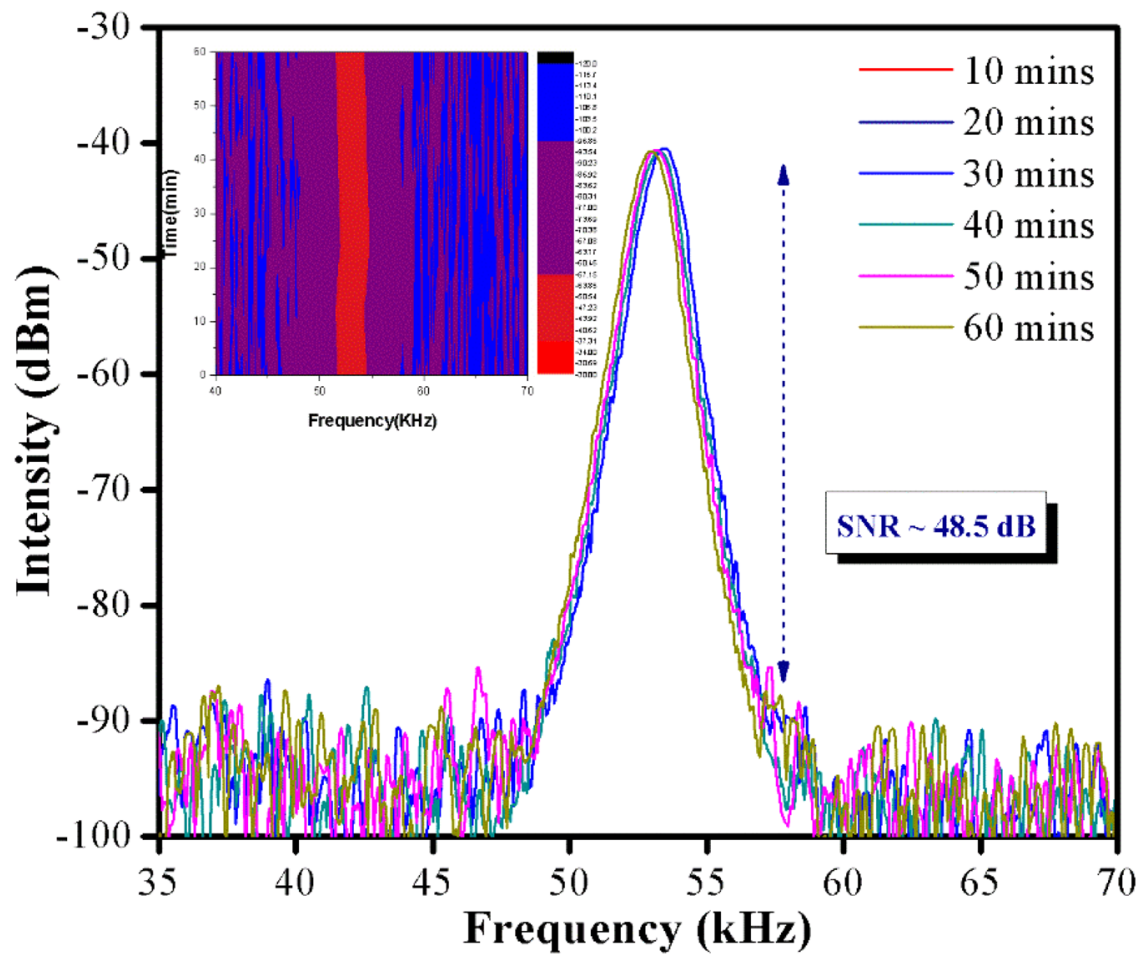

Figure 6. Stability performance of the passively Q-switched EDF laser within 60 minutes recorded using RFSA at a constant pump power of $221 \mathrm{~mW}$. 

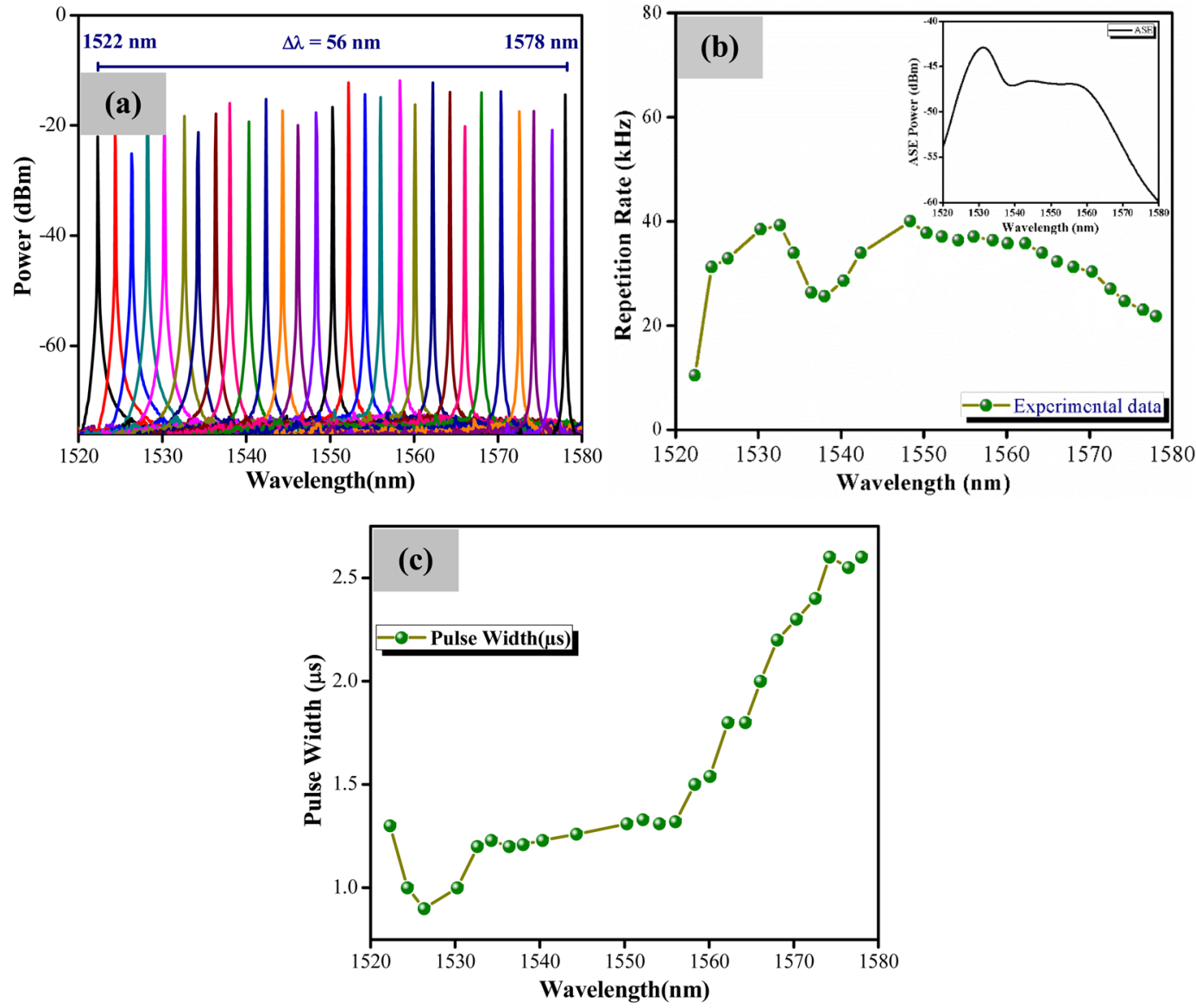

Figure 7. (a) Superimposed optical spectra of the tunable EDF laser at different wavelengths, (b) the repetition rate of Q-switching against the tunable lasing wavelength at constant pump power of $194.2 \mathrm{~mW}$ (Inset: ASE spectrum), and (c) the pulse width versus lasing wavelength.

\begin{tabular}{|c|c|c|c|c|c|c|c|}
\hline Saturable absorber & $\begin{array}{l}\text { Operation } \\
\text { wavelength } \\
(\mathrm{nm})\end{array}$ & $\begin{array}{c}\text { Tunable } \\
\text { wavelength } \\
\text { range ( } \mathrm{nm})\end{array}$ & $\begin{array}{l}\text { Pump power } \\
\text { range (mW) }\end{array}$ & $\begin{array}{l}\text { Pulse width } \\
(\mu \mathrm{s})\end{array}$ & $\begin{array}{l}\text { Repetition rate } \\
(\mathrm{kHz})\end{array}$ & $\begin{array}{l}\text { Maximum } \\
\text { pulse energy } \\
\text { (nJ) }\end{array}$ & Ref. \\
\hline $\mathrm{MoSe}_{2}-\mathrm{PVA}$ & 1560 & - & $570-720$ & $4.04-6.506$ & 60.724-66.847 & 369.5 & 52 \\
\hline $\mathrm{WSe}_{2}$ - PVA & 1560 & - & $280-720$ & $4.063-9.182$ & $46.281-85.365$ & 484.8 & 52 \\
\hline $\mathrm{MoWSe}_{2}$ & 1554 & - & $99-245$ & 6.80-1.90 & $26-48$ & 11.80 & 53 \\
\hline $\mathrm{MoS}_{2}$ - PVA & 1565 & - & $17.40-134.30$ & $23.20-5.40$ & $6.50-27$ & 63.20 & 30 \\
\hline $\mathrm{MoS}_{2}-\mathrm{PVA}$ & 1560 & $1519.6-1567.7$ & $18.9-227.1$ & $26.7-3.3$ & $8.77-43.47$ & 160 & 54 \\
\hline LPE Chitosan/MoS & 1561.5 & $1510-1580$ & $135.4-280.5$ & $1.68-1.02$ & $57.3-79.4$ & 43.69 & 55 \\
\hline WSSe & 1568.4 & $1530-1570$ & $89.07-280.5$ & $4.16-2.6$ & $27.52-61.81$ & 7.31 & 56 \\
\hline $\mathrm{MoSe}_{2}$-PVA & 1562 & - & $22.4-102.0$ & \begin{tabular}{|l|}
$59.1-30.4$ \\
\end{tabular} & $16.9-32.8$ & 57.9 & 57 \\
\hline $\mathrm{WTe}_{2}$ & 1531 & - & $212-630$ & $\sim 2.3-0.583$ & $144.7-240$ & 58.625 & 58 \\
\hline SnS & 1560 & - & $275-500$ & - & $36.36-65.19$ & - & 59 \\
\hline $\mathrm{BP}$ & 1988 & - & - & 1.78 & 19.25 & 7840 & 60 \\
\hline $\mathrm{WTe}_{2}$ - PVA & 1560.5 & $1522-1578$ & $124.9-244.5$ & $3.67-1.77$ & $38.39-55.56$ & 18.09 & This work \\
\hline
\end{tabular}

Table 1. Passively Q-switched fiber laser operating at $1.5 \mu \mathrm{m}$ by different $S A s$. $\mathrm{MoS}_{\mathrm{e}}=$ Molybdenum disulfide, $\mathrm{MoSe}_{2}=$ Molybdenum diselenide, WSe ${ }_{2}=$ Tungsten diselenide, MoWSe$_{2}=$ Molybdenum tungsten diselenide, WSSe $=$ Tungsten sulfide selenide, $\mathrm{SnS}=$ Tin Sulfide, $\mathrm{BP}=$ Black phosphorus.

film based SA generates Q-switched pulses with the narrowest pulse width. Furthermore, the threshold pump power for Q-switching to occur in this system is lower compared other similar systems ${ }^{29}$. Overall, the output performance of the proposed laser system is comparable and at some points is better than that in previous reports, thus confirming the applicability of $\mathrm{WTe}_{2}$ as an SA for Q-switched pulse generation at the C-band region. The excellent photoresponse behaviour possessed by tellurium $(\mathrm{Te})^{51}$ allows for good pulsed laser performance to the realized. 


\begin{abstract}
Methods
Preparation of a Tungsten Ditelluride (WTe2) - Based SA. Solution casting is used to fabricate the $\mathrm{WTe}_{2}$ film with a polyvinyl alcohol (PVA) polymer thin film host. The $\mathrm{WTe}_{2}$ solution is purchased from 2D Semiconductors at $99.99 \%$ purity while the PVA at MW 31,000 powder is obtained from Sigma Aldrich. Approximately $100 \mathrm{mg}$ of the PVA powder is slowly added into $10 \mathrm{ml}$ of deionized water (DIW) at $60^{\circ} \mathrm{C}$ and stirred continuously for 2 hours using a magnetic stirrer. A homogeneous $\mathrm{WTe}_{2}$ solution is obtained by treating the purchased $\mathrm{WTe}_{2}$ solution with a bath sonicator for a period of 30 minutes. Approximately $2 \mathrm{~mL}$ of the homogeneous $1 \mathrm{mg} / \mathrm{mL} \mathrm{WTe}{ }_{2}$ solution is added drop by drop into a beaker containing $8 \mathrm{~mL}$ of the $10 \mathrm{mg} / \mathrm{mL}$ PVA solution while stirring. The mixture is stirred continuously for another 15 minutes at $60^{\circ} \mathrm{C}$. Finally, the mixture is poured into a glass petri dish and heated in an oven for 2 hours at $60^{\circ} \mathrm{C}$, and the $\mathrm{WTe}_{2} / \mathrm{PVA}$ film is carefully removed from the petri dish after being allowed to cool down to room temperature.
\end{abstract}

Laser characterization. Analysis of the sample signal is done using the Yokogawa AQ6370C OSA as well as a Yokogawa DLM2054 oscilloscope (OSC) with a $1 \mathrm{GHz}$ photodetector. An Anritsu MS2683A radio frequency spectrum analyzer (RFSA) and a Thorlabs optical power meter (OPM) are used to monitor the output spectra for further analysis.

\title{
Conclusion
}

In this work, a broadband WTe 2 based SA is demonstrated for the passive generation of Q-switched pulses in the C-band region. A stable Q-switched output is achieved at a threshold pump power of $124.9 \mathrm{~mW}$ with a central wavelength of $1560.5 \mathrm{~nm}$. By increasing the pumping power from $124.9 \mathrm{~mW}$ to $244.5 \mathrm{~mW}$, the repetition rate rises from $38.39 \mathrm{kHz}$ to $55.56 \mathrm{kHz}$ while the pulse width decreases from $3.67 \mu$ s to $1.77 \mu$ s. The proposed laser system exhibits wide-band tunability of up to $56 \mathrm{~nm}$ from $1522 \mathrm{~nm}$ to $1578 \mathrm{~nm}$, and is highly stable with no significant fluctuation in frequency observed over an operation period of 60 minutes with average SNR values of $48.5 \mathrm{~dB}$. The obtained experimental results imply that $\mathrm{WTe}_{2}$ has a great potential as SA and the proposed laser system can be used as a pulse tunable laser source for various optical telecommunications and measurement applications.

Received: 27 November 2019; Accepted: 8 January 2020;

Published online: 17 June 2020

\section{References}

1. Xing, C. et al. 2D Nonlayered selenium nanosheets: Facile synthesis, photoluminescence, and ultrafast photonics. Advanced Optical Materials 5, 1700884 (2017).

2. Ahmad, H., Albaqawi, H. S., Yusoff, N., Chong, W. Y. \& Yasin, M. Q-Switched fiber laser at $1.5 \mu \mathrm{m}$ region using $\mathrm{Ti}_{3} \mathrm{AlC}_{2} \mathrm{MAX}_{\mathrm{Phase}}$ based saturable absorber. IEEE Journal of Quantum Electronics (2019).

3. Wang, Y. et al. An all-optical, actively Q-switched fiber laser by an antimonene-based optical modulator. Laser \& Photonics Reviews 13, 1800313 (2019).

4. Xu, Y. et al. Solvothermal synthesis and ultrafast photonics of black phosphorus quantum dots. Advanced Optical Materials 4, 1223-1229 (2016).

5. Zhao, H. et al. Stable pulse-compressed acousto-optic Q-switched fiber laser. Optics Letters 32(19), 2774-2776 (2007).

6. El-Sherif, A. F. \& King, T. A. High-energy, high-brightness Q-switched $\mathrm{Tm}^{3+}$-doped fiber laser using an electro-optic modulator. Optics Communications 218(4), 337-344 (2003).

7. Ahmad, H., Reduan, S., Yusoff, N., Ismail, M. \& Aidit, S. Mode-locked pulse generation in erbium-doped fiber laser by evanescent field interaction with reduced graphene oxide-titanium dioxide nanohybrid. Optics \& Laser Technology 118, 93-101 (2019).

8. Ismail, M., Ahmad, F., Harun, S. W., Arof, H. \& Ahmad, H. A Q-switched erbium-doped fiber laser with a graphene saturable absorber. Laser Physics Letters 10, 025102 (2013).

9. Cheng, L. et al. Linear and nonlinear optical properties modulation of $\mathrm{Sb}_{2} \mathrm{Te}_{3} / \mathrm{GeTe}$ bilayerfilm as a promising saturable absorber. Results in Physics 13, 102282 (2019).

10. Ahmad, H. et al. Tunable S-band Q-switched fiber laser using $\mathrm{Bi}_{2} \mathrm{Se}_{3}$ as the saturable absorber. IEEE Photonics J. 7(3), 1-8 (2015).

11. Lee, J. et al. Linearly polarized, Q-switched, erbium-doped fiber laser incorporating a bulk-structured bismuth telluride/polyvinyl alcohol saturable absorber. Opt. Eng. 55(7), 076109 (2016).

12. Zhou, Y. et al. Recent advances in black phosphorus-based photonics, electronics, sensors and energy devices. Materials Horizons 4 , 997-1019 (2017).

13. Guo, Z. et al. From black phosphorus to phosphorene: basic solvent exfoliation, evolution of Raman scattering, and applications to ultrafast photonics. Advanced Functional Materials 25, 6996-7002 (2015).

14. Li, P. et al. Two-dimensional $\mathrm{CH}_{3} \mathrm{NH}_{3} \mathrm{PbI}_{3}$ perovskite nanosheets for ultrafast pulsed fiber lasers. ACS Applied Materials \& Interfaces 9, 12759-12765 (2017).

15. Zhou, D. P., Wei, L., Dong, B. \& Liu, W. K. Tunable passively Q-switched erbium-doped fiber laser with carbon nanotubes as a saturable absorber. IEEE Photonics Technol. Lett. 22(1), 9-11 (2010).

16. Popa, D., Sun, Z., Hasan, T., Torrisi, F., Wang, F. \& Ferrari, A. C. Graphene Q-switched, tunable fiber laser. Appl.Phys. Lett. 98(7), $073106(2011)$.

17. Wu, L. et al. Few-layer tin sulfide: A promising black-phosphorus-analogue 2D material with exceptionally large nonlinear optical response, high stability, and applications in all-optical switching and wavelength conversion. Advanced Optical Materials 6, 1700985 (2018).

18. Zhang, Y. et al. Photonics and optoelectronics using nano-structured hybrid perovskite media and their optical cavities. Physics Reports (2019).

19. Tao, W. et al. Emerging two-dimensional monoelemental materials (Xenes) for biomedical applications. Chemical Society Reviews (2019).

20. Zhang, H. N. \& Gold, J. L. nanobipyramids as saturable absorbers for passively Qswitched laser generation in the $1.1 \mathrm{~lm}$ region. Optics Lett. 41, 1150-1152 (2016).

21. Plamann, K. et al. Ultrashort pulse laser surgery of the cornea and the sclera. Journal of Optics 12(8), 084002 (2010).

22. Stöppler, G., Kieleck, C. \& Eichhorn, M. High-pulse energy Qswitched $\mathrm{Tm}^{3+}$ : YAG laser for nonlinear frequency conversion to the mid-IR. In Security+ Defence. International Society for Optics and Photonics (pp. 783609-783609) (2010).

23. Kölbl, J., Fröschl, M., Seedsman, A. \& Sperber, P. Near-infrared laser range finder using kHz repetition rate. In SPIE Europe Security and Defence. International Society for Optics and Photonics (pp. 71150M-71150M) (2008). 
24. Kilpela, A., Pennala, R. \& Kostamovaara, J. Precise pulsed time-of-flight laser range finder for industrial distance measurements. Review of Scientific Instruments 72(4), 2197-2202 (2001).

25. Vicente, S. C., Gamez, M. M., Kir'yanov, A. V., Barmenkov, Y. O. \& Andres, M. V. Diode-pumped self-Q-switched erbium-doped all-fibre laser. Quantum Electronics 34(4), 310 (2004).

26. Song, Y., Shi, X., Wu, C., Tang, D. \& Zhang, H. Recent progress of study on optical solitons in fiber lasers. 6, 021313 (2019).

27. Nady, A. et al. Nickel oxide nanoparticles as a saturable absorber for an all-fiber passively Q-switched erbium-doped fiber laser. Laser Phys. 27, 065105 (6pp) (2017).

28. Woodward, R. I. \& Kelleher, E. J. R. D. S. A. f. F. L. Appl. Sci. 5, 1440-1456 (2015).

29. Mohanraj, J., Velmurugan, V. \& Sivabalan, S. Transition metal dichalcogenides based saturable absorbers for pulsed laser technology. Optical Materials 60, 601-617 (2016).

30. Luo, Z. et al. 1-, 1.5-, and 2- $\mu \mathrm{m}$ fiber lasers Q-switched by a broadband few-layer $\mathrm{MoS}_{2}$ saturable absorber. J. Lightwave Technol. 32, 4077-4084. (2014).

31. Zhang, M. et al. Yb-and Er-doped fiber laser Q-switched with an optically uniform, broadband $\mathrm{WS}_{2}$ saturable absorber. Sci. Rep. 5, 17482-17491 (2015).

32. Liu, Y. et al. Photoresponsivity of an all-semimetal heterostructure based on graphene and WTe ${ }_{2}$. Scientific Reports $\mathbf{8}, 12840$ (2018).

33. Torun, E., Sahin, H., Cahangirov, S., Rubio, A. \& Peeters, F. Anisotropic electronic, mechanical, and optical properties of monolayer $\mathrm{WTe}_{2}$. Journal of Applied Physics 119, 074307 (2016).

34. Zheng, F. et al. On the Quantum Spin Hall Gap of Monolayer 1T'-WTe 2 . Advanced Materials 28, 4845-4851 (2016).

35. Eftekhari, A. Tungsten dichalcogenides $\left(\mathrm{WS}_{2}, \mathrm{WSe}_{2}\right.$, and $\left.\mathrm{WTe}_{2}\right)$ : Materials chemistry and applications. Journal of Materials Chemistry A 5, 18299-18325 (2017).

36. Ghosh, B., Gupta, A. \& Bishnoi, B. Effects of defects on the electronic properties of $\mathrm{WTe}_{2}$ armchair nanoribbons. Journal of Semiconductors 35, 113002 (2014).

37. Woodward, R. I. \& Kelleher, E. J. 2D saturable absorbers for fibre lasers. Applied Sciences 5, 1440-1456 (2015).

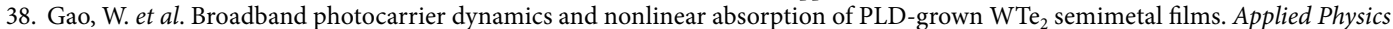
Letters 112, 171112 (2018).

39. Ali, M. N. et al. Large, non-saturating magnetoresistance in $\mathrm{WTe}_{2}$. Nature 514, 205 (2014).

40. Wang, J. et al. Magnetron-sputtering deposited $\mathrm{WTe}_{2}$ for an ultrafast thulium-doped fiber laser. Optics Letters 42, 5010-5013 (2017).

41. Koo, J. et al. Near-infrared saturable absorption of defective bulk-structured $\mathrm{WTe}_{2}$ for femtosecond laser mode-locking. Advanced Functional Materials 26, 7454-7461 (2016).

42. Kim, Y. et al. Anomalous lattice dynamics of mono-, bi-, and tri-layer $\mathrm{WTe}_{2}$. arXiv preprint arXiv:1508.03244 (2015).

43. Lee, C.-H. et al. Tungsten ditelluride: a layered semimetal. Scientific Reports 5, 10013 (2015).

44. Woodward, R. et al. Tunable Q-switched fiber laser based on saturable edge-state absorption in fewlayer molybdenum disulfide $\mathrm{MoS}_{2}$. Opt. Express 22, 31113-31122 (2014).

45. Chen, Y. et al. Mechanically exfoliated black phosphorus as a new saturable absorber for both Q-switching and mode-locking laser operation. Optics Express 23, 12823-12833 (2015).

46. Luo, Z. et al. Two-dimensional material-based saturable absorbers: towards compact visible-wavelength all-fiber pulsed lasers. Nanoscale 8, 1066-1072 (2016).

47. Ko, S., Lee, J. \& Lee, J. H. Passively Q-switched ytterbium-doped fiber laser using the evanescent field interaction with bulk-like WTe, particles. Chin. Opt. Lett. 16, 020017 (2018).

48. Adel, P. Pulsed fiber lasers. (Cuvillier Verlag, 2004).

49. Ahmad, H., Ismail, M. \& Aidit, S. Optically modulated tunable O-band Praseodymium-doped fluoride fiber laser utilizing multiwalled carbon nanotube saturable absorber. Chinese Physics Letters 36, 104202 (2019).

50. Ahmad, H. et al. Tunable Q-switched erbium-doped fiber laser in the C-band region using nanoparticles $\left(\mathrm{TiO}_{2}\right)$. Optics Communications 435, 283-288 (2019).

51. Xie, Z. et al. Ultrathin 2D nonlayered tellurium nanosheets: Facile liquid-phase exfoliation, characterization, and photoresponse with high performance and enhanced stability. Advanced Functional Materials 28, 1705833 (2018).

52. Chen, B. et al. Q-switched fiber laser based on transition metal dichalcogenides $\mathrm{MoS}_{2}, \mathrm{MoSe}_{2}, \mathrm{WS}_{2}$, and $\mathrm{WSe}_{2}$. Optics Express 23, 26723-26737 (2015).

53. Ahmad, H. et al. Ternary MoWSe ${ }_{2}$ alloy saturable absorber for passively Q-switched Yb-, Erand Tm-doped fiber laser,. Optics Communications 437, 355-362 (2019).

54. Huang, Y. et al. Opt. Express 22, 25258 (2014).

55. Ahmad, H., Aidit, S. N., Ooi, S. I. \& Tiu, Z. C. Tunable passively Q-switched erbium-doped fiber laser with Chitosan/MoS 2 saturable absorber. Optics and Laser Technology 103, 199-205 (2018).

56. Ahmad, H., Tiu, Z. C. \& Ooi, S. I. Passive Q-switching in an erbium-doped fiber laser using tungsten sulphoselenide as a saturable absorber. Chinese Optics Letters 16(2), 020009 (2018).

57. Ahmad, H. M. S., Z. C. Tiu, A. Zarei, and S. W. Harun. Opt. Laser Technol. 79, 20 (2016).

58. Liu, M., Ouyang, Y., Hou, H., Liu, W. \& Wei, Z. Q-switched fiber laser operating at $1.5 \mu$ mbased on WTe 2 . Chinese Optics Letters 17(2), 020006 (2019).

59. Xie, Z. et al. Revealing of the ultrafast third-order nonlinear optical response and enabled photonic application in two-dimensional tin sulfide. Photonics Research 7, 494-502 (2019).

60. Chu, Z., Liu, J., Guo, Z. \& Zhang, H. $2 \mu$ m passively Q-switched laser based on black phosphorus. Opt. Mater. Express 6, 2374-2379 (2016).

\section{Acknowledgements}

We would like to thank the University of Malaya for funding this research under the grants BKS002 - 2019, RK021 - 2019 and RU011 - 2019 and the Ministry of Higher Education, Malaysia for funding this research under the grant HiCoE Phase II Funding.

\section{Author contributions}

Harith Ahmad proposed the study and designed the experiment. Hissah Saedoon Albaqawi performed the fiber laser experiments and wrote the manuscript. Norazriena Yusoff prepared the $\mathrm{WTe}_{2}$ films, performed the material characterization and contributed to writing of the manuscript. Chong $\mathrm{Wu}$ Yi contributed to data analysis.

\section{Competing interests}

The authors declare no competing interests. 
Additional information

Correspondence and requests for materials should be addressed to H.A.

Reprints and permissions information is available at www.nature.com/reprints.

Publisher's note Springer Nature remains neutral with regard to jurisdictional claims in published maps and institutional affiliations.

(c) (i) Open Access This article is licensed under a Creative Commons Attribution 4.0 International License, which permits use, sharing, adaptation, distribution and reproduction in any medium or format, as long as you give appropriate credit to the original author(s) and the source, provide a link to the Creative Commons license, and indicate if changes were made. The images or other third party material in this article are included in the article's Creative Commons license, unless indicated otherwise in a credit line to the material. If material is not included in the article's Creative Commons license and your intended use is not permitted by statutory regulation or exceeds the permitted use, you will need to obtain permission directly from the copyright holder. To view a copy of this license, visit http://creativecommons.org/licenses/by/4.0/.

(C) The Author(s) 2020 